\title{
Recent Developments in Quantitative Electron Tomography
}

\author{
P.A. Midgley*, Z. Saghi, ${ }^{*}$ J. Hindson, ${ }^{* *}$ R. Leary, ${ }^{*}$ D.J. Holland,*** A.J. Sederman, ${ }^{* * *}$ L.F. \\ Gladden, $* * *$ V. Schmidt, $* * * *$ and N. Greenham** \\ * Department of Materials Science and Metallurgy, University of Cambridge, Pembroke Street, \\ Cambridge, CB2 3QZ, UK. \\ ** Cavendish Laboratory, University of Cambridge, JJ Thompson Avenue, Cambridge, CB3 0HE, \\ UK \\ *** Department of Chemical Engineering and Biotechnology, University of Cambridge, Pembroke \\ Street, Cambridge, CB2 3RA, UK. \\ **** Institute of Stochastics, Ulm University, D-89069 Ulm, Germany.
}

Electron tomography has matured over the past decade to become a technique used in both the life sciences and materials science almost on a routine basis. In materials science, the ability of most samples to withstand relatively large electron doses has seen the development of many tomographic imaging modes. The ubiquitous nature of bright-field imaging has led to its use in electron tomography across many fields of materials science, but especially in the study of soft matter where dynamical effects and diffraction contrast is often minimal [1]. For many materials however, STEM HAADF tomography remains the mode of choice, yielding high contrast images which are relatively free of crystallographic artefacts [2]. A combination of STEM tomography and EELS has seen the first examples of 3D chemical mapping [3] and, similarly, EFTEM tomography can produce volume-specific compositional information [4]. Defects and secondary phases can be mapped using diffraction contrast tomography, if the diffraction conditions do not change significantly across the tilt series [5]. Recent work has shown that time-resolved electron tomography is possible, with femtosecond pulsing of nanostructures showing vibrational characteristics in 3D [6]. With all these modes, however, there remains a pressing need to extract reliable, quantitative information from 3D reconstructions. This information can be subject to many sources of error, at image acquisition, during the reconstruction process, through to segmentation, visualization and during additional tomogram processing. In order to be more confident in the information garnered from tomograms we need methods to improve the fidelity of the reconstruction (e.g. minimizing missing wedge artefacts) and more automated and objective ways to best process the tomograms to recover key information.

Reconstruction. We have initiated recently a project of work to determine methods to apply additional constraints in the reconstruction process. Although SIRT has become a popular reconstruction method it is well known that after perhaps 30-40 iterations the apparent match between the reconstruction and the original set of images can get progressively worse. One possible explanation is that the method does not take into account the fact that the re-projected image (from the tomogram) has a better signal-to-noise ratio than the original image. This mismatch in noise content is not accounted for in conventional SIRT routines and can lead to a divergence away from the 'best' solution. Recent work [7] studying the SIRT method has shown that smaller objects tend to be reconstructed less well than larger objects in the tomogram. In a similar vein, Norton [8] has shown that a larger number of iterations are required for objects with proportionally higher spatial frequencies. Such imperfections have led a number of groups to consider alternative reconstruction methods. 
One of these, discrete tomography [9], has in recent years been successful in improving the quality of the reconstruction by incorporating a priori knowledge about the sample. This has been used in two ways: either that the object itself is made up of discrete entities (for example atoms in a nanoparticle) or that the object has a discrete number of densities (i.e. a tomographic slice would be composed of a discrete number of grey levels). This ability to build in prior information is also true for compressed sensing (CS) approaches to tomography [10]. Here a transform is used to convert the set of images to a space that is 'sparse', i.e. in which there are relatively few non-zero pixels. The reconstruction then becomes an optimization problem where minimizing the number of non-zero pixels is the objective. This can be solved by minimizing the $l_{1}$ norm. Thus, if one can transform the set of images into a sparse space, this approach should be successful at improving the reconstruction. Discrete tomography can be thought of as related to CS by considering the grey levels, or discrete entities, as being 'sparse'. It seems possible to improve the reconstruction further by taking into account the 'total variation' of each image. Minimizing this appears to help to reduce the effects of noise in the tomogram. In this paper we will highlight how this approach can be used on typical materials tomographic data sets where the constraint of sparseness is satisfied.

Tomogram Processing. Once the tomogram is reconstructed there is often a pressing need to extract information in an automated, objective and unbiased fashion. Many of the samples studied with electron tomography are nanoscale particles (rods, spheres, etc) which appear either in isolation or as part of a composite object. In these cases, we can use a new approach and describe these objects in terms of spheres whose radii and positions are known accurately in 3D [11]. This approach requires individual particles to be identified in the tomogram (using a number of image processing steps) and identifying sphere positions and sizes that best match the objects in the tomogram. Once that best-fit is achieved, the size and co-ordinates of the spheres can be used for a variety of statistical analyses. In particular, we have applied this approach recently to a study of a novel solar cell material composed of $\mathrm{CdSe}$ nanoparticles dispersed in a $\mathrm{OC}_{1} \mathrm{C}_{10} \mathrm{PPV}$ polymer matrix [12]. After the extraction of spheres, neighbouring particles can be connected to form a network whose spatial distribution, in this case, can be related to the transport of electrons through the solar cell [13].

References

[1] H. Jinnai and R.J. Spontak Polymer 50 (2008) 1067

[2] P.A. Midgley and M. Weyland Ultramicroscopy 96 (2003) 413

[3] K. Jarausch et al. Ultramicroscopy 109 (2009) 326

[4] M.H. Gass et al. Nano Lett. 6 (2006) 376

[5] J.S. Barnard et al. Phil. Mag. 86 (2006) 4901

[6] O.H. Kwon and A. Zewail Science 328 (2010) 1668

[7] C. Kuebel et al. Microsc Microanal 15 (Suppl 2) (2009) 602

[8] S.J. Norton J. Opt. Soc. Am. A 2, (1985) 6

[9] K.J. Batenburg et al. Ultramicroscopy 109 (2009) 730.

[10] M. Lustig et al Magnetic Resonance in Medicine 58 (2007) 1182

[11] R. Thiedmann et al. (2011) submitted to J. Mathematical Imaging and Vision

[12] J.C. Hindson et al Nano Lett. 11 (2011) 904

[13] The authors gratefully acknowledge R. Thiedmann, A. Spettl, O. Stenzel and T. Zeibig. The work was supported by the EPSRC, U.K., and by the European Union under the Framework 6 program under a contract for an Integrated Infrastructure Initiative, Reference 026019 ESTEEM. 